Communication brève

\title{
Syndrome de Sweet induit par le pegfilgrastim au cours d'un syndrome myélodysplasique AREB2 : à propos d'un cas clinique
}

\section{Sweet's syndrome induced by pegfilgrastim during a myelodysplastic syndrome} AREB2: A case report

\author{
S. Nelis ${ }^{\mathrm{a}, *}$, M.-A. Azerad ${ }^{\mathrm{b}}$, A. Drowart ${ }^{\mathrm{c}}$, P. Lewalle ${ }^{\mathrm{d}}$, A. Efira $^{\mathrm{e}}$ \\ a Service de rhumatologie - médecine physique, hôpital Erasme, 808, route de Lennik, 1070 Anderlecht, Belgique \\ ${ }^{\mathrm{b}}$ Service d'hématologie, CHR de la Citadelle, 1, boulevard du $12^{e}$ de Ligne, 4000 Liège, Belgique \\ c Service d'oncologie médicale, institut Jules-Bordet, 121-125, boulevard de Waterloo, 1000 Bruxelles, Belgique \\ d Service d'hématologie, institut Jules-Bordet, 121-125, boulevard de Waterloo, 1000 Bruxelles, Belgique \\ e Service d'hématologie-oncologie, CHU Brugmann, 4, place Arthur Van Gehuchten, 1020 Bruxelles, Belgique
}

\section{N F O A R T I C L E}

\section{Historique de l'article :}

Disponible sur Internet le 11 décembre 2018

\section{Mots clés :}

Syndrome de Sweet

Dermatose neutrophilique aiguë

Syndrome myélodysplasique

G- CSF

Pegfilgrastim

\section{R É S U M É}

Introduction. - Le syndrome de Sweet est une dermatose neutrophilique aiguë caractérisée par l'apparition brutale de lésions cutanées accompagnées de fièvre, d'arthralgies, d'une leucocytose et d'une infiltration neutrophilique diffuse du derme, ainsi que par une excellente réponse à la corticothérapie.

Observation. - Un patient de 46 ans, ayant un syndrome myélodysplasique, était hospitalisé pour une chimiothérapie. Au $8^{\mathrm{e}}$ jour de la chimiothérapie, il recevait une dose unique de pegfilgrastim. Trois jours plus tard, il développait de la fièvre, une conjonctivite, des arthralgies et des lésions papulonodulaires érythémateuses et douloureuses. Une antibiothérapie empirique à large spectre était débutée mais le patient se dégradait. La biologie montrait une pancytopénie et un syndrome inflammatoire. Les bilans microbiologique, auto-immun et le scanner thoracique revenaient négatifs. La biopsie cutanée était compatible avec un syndrome de Sweet. Un diagnostic de syndrome de Sweet sur pegfilgrastim était retenu et une corticothérapie intraveineuse était débutée avec une guérison rapide.

Conclusion. - La survenue d'un syndrome de Sweet est une complication rare des traitements par G-CSF.

(c) 2018 Publié par Elsevier Masson SAS au nom de Société Nationale Française de Médecine Interne

(SNFMI).

\section{A B S T R A C T}

Introduction. - Sweet's syndrome is an acute neutrophilic dermatosis characterized by abrupt onset of skin lesions accompanied by fever, arthralgia, leukocytosis and diffuse neutrophilic infiltration of the dermis, as well as an excellent response to corticosteroid therapy.

Case report. - A 46-year-old patient with myelodysplastic syndrome was admitted for chemotherapy. On the eighth day of chemotherapy, he received a single dose of pegfilgrastim. Three days later, he developed pyrexia, conjunctivitis, arthralgia and erythematous and painful papulo-nodular lesions. Broad-spectrum empiric antibiotic therapy was started but the patient's condition deteriorated. Biology showed pancytopenia and inflammatory syndrome. Microbiological tests, autoimmune serologies and chest-computed tomography were negative. Cutaneous biopsy was compatible with Sweet's syndrome. A diagnosis of Sweet's syndrome induced by pegfilgrastim was made and intravenous corticosteroid therapy was started with a rapid favorable outcome.

Conclusion. - Sweet's syndrome is a rare adverse effect of G-CSF.

(C) 2018 Published by Elsevier Masson SAS on behalf of Société Nationale Française de Médecine Interne (SNFMI)

\footnotetext{
* Auteur correspondant.

Adresse e-mail : sabbeke32@hotmail.be (S. Nelis).
} 


\section{Introduction}

Le syndrome de Sweet est une dermatose neutrophilique aiguë caractérisée par l'apparition brutale de lésions cutanées accompagnées de fièvre, d'arthralgies, d'une leucocytose et d'une infiltration neutrophilique diffuse du derme, ainsi que par une excellente réponse à la corticothérapie.

\section{Observation}

Un homme de 46 ans, d'origine marocaine, était hospitalisé dans le service d'hématologie, pour une chimiothérapie d'induction en vue d'une allogreffe de moelle. Le patient était atteint d'un syndrome myélodysplasique AREB-1, développé sur un clone d'hémoglobinurie paroxystique nocturne, qui avait récemment évolué en syndrome myélodysplasique AREB-2. Il n'avait pas d'autres antécédents médicaux. Son traitement à domicile comprenait de l'acyclovir, de la ciprofloxacine et du fluconazole prophylactiques en raison d'une neutropénie chronique. Son examen physique à l'admission était banal, hormis la palpation d'une splénomégalie.

Au $8^{\mathrm{e}}$ jour de la chimiothérapie (cytarabine, daunorubicine), il recevait une dose unique de pegfilgrastim (Neulasta) à la dose de $6 \mathrm{mg}$ en sous-cutané. Trois jours plus tard, le patient présentait une fièvre à $38,5^{\circ} \mathrm{C}$, des arthralgies diffuses des grosses articulations, une conjonctivite et une éruption cutanée papulonodulaire érythémateuse et douloureuse de la paupière inférieure droite, des coudes, des avant-bras, du dos des mains et des jambes (Fig. 1-3). Les diagnostics différentiels de cette éruption cutanée fébrile chez ce patient incluaient l'infection, l'hypersensibilité médicamenteuse, l'érythème polymorphe, les vascularites au sens

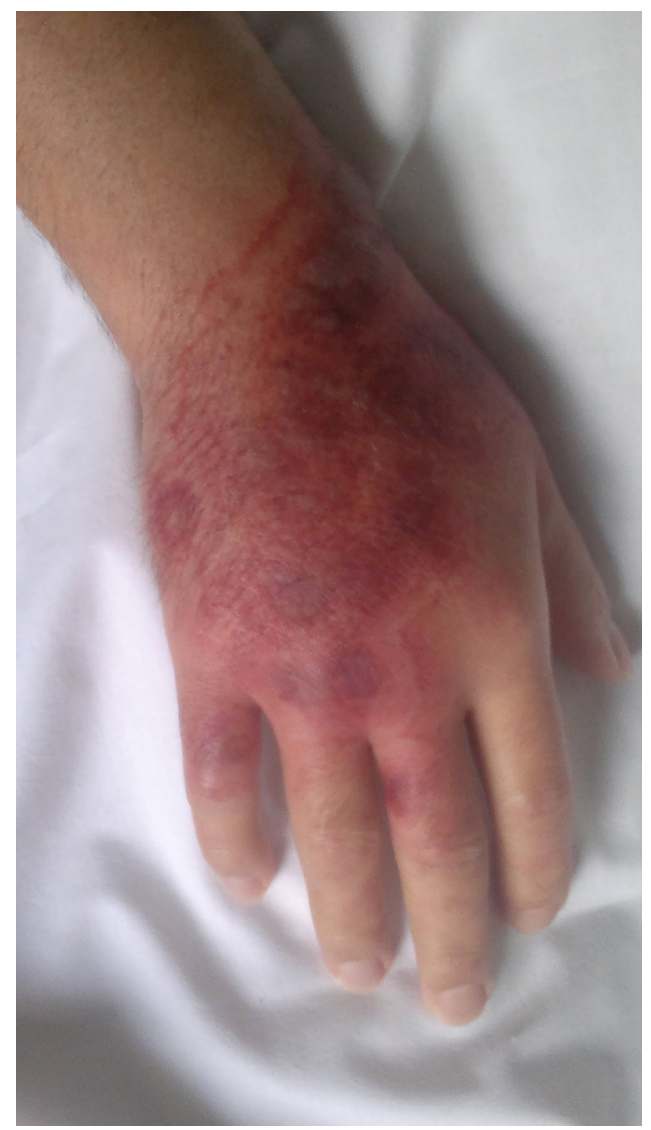

Fig. 1. Macules érythémateuses et violacées, coalescentes en une plaque, au niveau du poignet et du dos de la main droite.

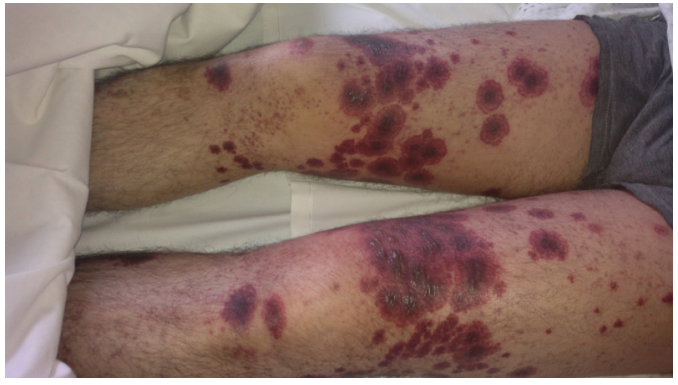

Fig. 2. Lésions papulonodulaires érythémateuses et violacées, de forme annulaire, au niveau des cuisses.

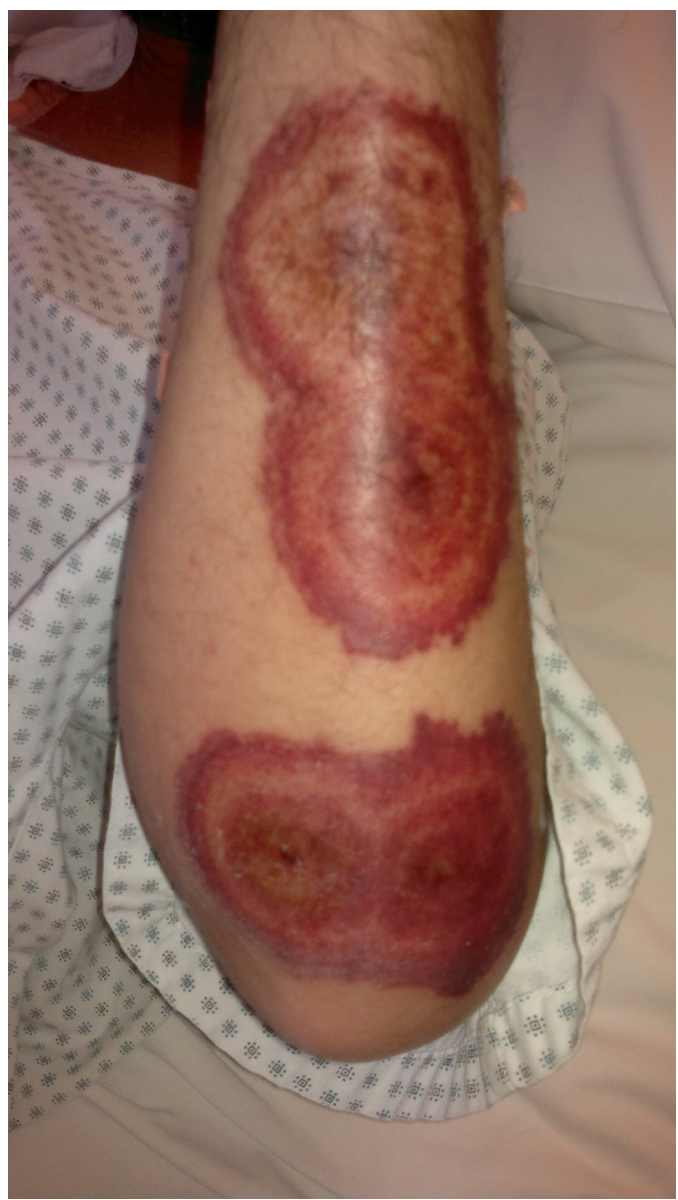

Fig. 3. Plaques érythémateuses, de forme annulaire, au niveau de l'avant-bras gauche.

large et le syndrome de Sweet (SS). La biologie montrait une pancytopénie avec une anémie normocytaire normochrome à $8,1 \mathrm{~g} / \mathrm{dl}$, une leucopénie à $1550 / \mathrm{mm}^{3}$ avec une neutropénie à $350 / \mathrm{mm}^{3}$, une thrombopénie à $16000 / \mathrm{mm}^{3}$, une élévation de la CRP à $200 \mathrm{mg} / \mathrm{L}$ et une vitesse de sédimentation à $31 \mathrm{~mm} / \mathrm{h}$.

Afin de couvrir une origine infectieuse, le patient était traité empiriquement par pipéracilline-tazobactam en association à la vancomycine, en ajout de l'acyclovir et du fluconazole et l'administration de ciprofloxacine était suspendue. Après 48 heures de traitement empirique, les lésions cutanées s'étendaient, devenaient plus violacées et nécrotiques et le patient continuait à être fébrile. Des hémocultures, un examen microscopique des urines, un prélèvement de gorge ainsi que des sérologies $H S V, V Z V, C M V$, EBV, HHV6, rougeole, rubéole, entérovirus, syphilis, Mycoplasme pneumoniae, Chlamydia pneumoniae, et l'antigène Aspergillus revenaient 


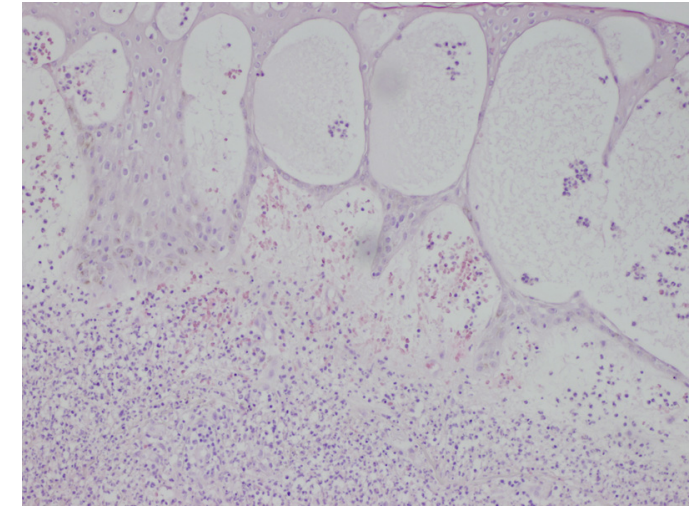

Fig. 4. Biopsie cutanée $(\times 20)$ montrant un épiderme hyperplasique, un œdème du derme papillaire, un infiltrat neutrophilique diffus du derme superficiel et moyen et l'absence de vascularite.

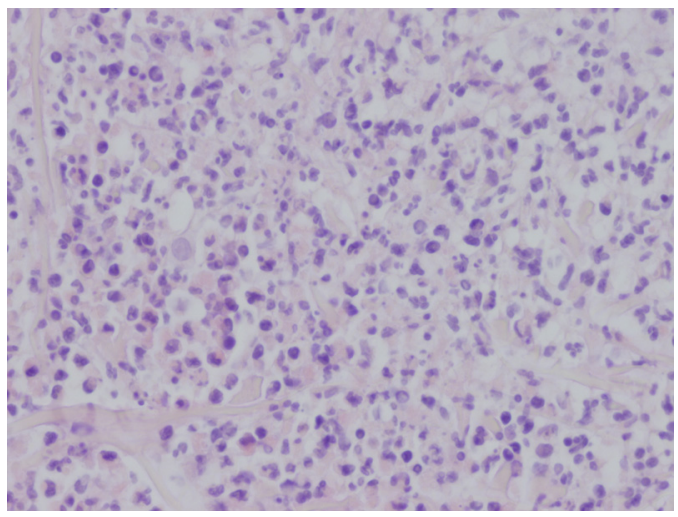

Fig. 5. Biopsie cutanée $(\times 40)$ montrant un infiltrat diffus neutrophilique au sein du derme.

négatifs. Le bilan auto-immun comprenant la recherche de FAN, ANCA, FR, anti-CCP, cryoglobuline et le complément était négatif. Le scanner thoracique ne montrait pas d'infiltrat pulmonaire.

Deux biopsies cutanées ont également été réalisées et montraient un infiltrat inflammatoire dense, constitué de neutrophiles, un œdème au niveau du derme papillaire et une extravasation de globules rouges formant de larges plages hémorragiques, sans signe de vascularite (Fig. 4 et 5). La culture tissulaire était négative. Le diagnostic de syndrome de Sweet sur pegfilgrastim était retenu et un traitement par méthylprednisolone en intraveineux à la dose de $1 \mathrm{mg} / \mathrm{kg}$ était débuté. L'amélioration était spectaculaire en 48 heures et en une semaine, les lésions cutanées disparaissaient totalement. La corticothérapie était poursuivie pendant un mois au total, à dose progressivement décroissante. Le pegfilgrastim n'a pas été re-administré au patient et celui-ci n'a pas présenté de récidive de SS par la suite.

\section{Discussion}

Le SS, encore appelé dermatose aiguë fébrile neutrophilique, a été décrit pour la première fois en 1964 par Robert Sweet [1]. Il apparaît à l'âge adulte et touche préférentiellement les femmes [1]. Le diagnostic est basé sur des critères cliniques et histopathologiques (Tableau 1) [2]. Il est caractérisé par une éruption cutanée brutale de plaques ou de nodules érythémateux douloureux, une altération de l'état général, une fièvre récurrente, des polyarthralgies et une hyperleucocytose neutrophilique. L'histologie montre un infiltrat mononuclée à prédominance neutrophilique, sans signe de vasculite leucocytoclasique, un exsudat hématique et un œdème dermique superficiel. Le traitement consiste en l'administration de
Tableau 1

Critères majeurs et mineurs pour le diagnostic de syndrome de Sweet classique [2].

Critères majeurs

Apparition brutale de plaques ou de nodules érythémateux douloureux évocateurs

Infiltrat dermique dense avec prédominance de polynucléaires neutrophiles sans vasculite leucocytoclasique

Critères mineurs

Fièvre $>38^{\circ} \mathrm{C}$

Précédée par une infection respiratoire ou gastro-intestinale ou associée à une maladie inflammatoire ou à un cancer (hémopathie ou tumeur solide) Excellente réponse à la corticothérapie générale ou à l'iodure de potassium Anomalies biologiques ( 3 anomalies sur 4 au moins)

VS $>20 \mathrm{~mm} / \mathrm{h}$

CRP augmentée

Leucocytes à $8000 / \mathrm{mm}^{3}$

Dont $>70 \%$ de PNN

Pour poser le diagnostic d'un syndrome de Sweet, il faut la présence de 2 critères majeurs et au moins 2 critères mineurs.

Tableau 2

Critères diagnostiques du syndrome de Sweet d'origine médicamenteuse [3].

Apparition brutale de plaques ou de nodules érythémateux douloureux

Preuve histopathologique d'une infiltration neutrophilique dense sans vasculite

Température $>38^{\circ} \mathrm{C}$

Relation entre l'administration du médicament et l'apparition des symptômes ou récidive des symptômes lors de la reprise du médicament

Résolution grâce à l'arrêt du médicament ou après traitement par corticostéroïdes

Les 5 critères doivent être présents pour poser le diagnostic d'un syndrome de Sweet d'origine médicamenteuse.

corticostéroïdes à la dose de $1 \mathrm{mg} / \mathrm{kg}$ de prednisolone avec une dégression progressive et une durée totale de 2 à 6 semaines. Il existe 4 catégories de SS :

- classique/idiopathique (le plus fréquent, parfois précédé par une infection respiratoire haute, soit associé à une pathologie intestinale inflammatoire ou à la grossesse) ;

- induit par les médicaments (surtout le G-CSF) ;

- paranéoplasique (surtout les hémopathies malignes);

- et la dermatose neutrophilique des mains [1].

Notre patient répond aux 5 critères diagnostiques d'un SS d'origine médicamenteuse (Tableau 2) [3].

Notons que les syndromes myélodysplasiques font partie des hémopathies malignes classiquement associées aux SS paranéoplasiques. Cependant, chez notre patient, la survenue du SS pouvait facilement être imputée à l'administration de pegfilgrastim plutôt qu'au syndrome myélodysplasique étant donné la chronologie de survenue du SS (3 jours après l'administration de pegfilgrastim).

Plusieurs cas de SS secondaires à l'administration de filgrastim (G-CSF) chez des patients neutropéniques ont été décrits, surtout en présence d'une hémopathie maligne, même si le syndrome de Sweet est généralement associé à une neutrophilie [1,4-9]. En particulier, sur une série rétrospective monocentrique récente de 83 SS, 8 cas de SS (10\% des patients) induits par le filgrastim ont été rapportés.

En revanche, l'apparition d'un syndrome de Sweet suite à l'administration du pegfilgrastim (G-CSF pégylé), n'a été que très peu décrit jusqu'à présent. À notre connaissance, il ne s'agit que du $4^{\mathrm{e}}$ cas de syndrome de Sweet après administration de pegfilgrastim [4-8].

La pathogenèse du SS est encore peu connue ; les cytokines IL-1, IL-8, G-CSF et GM-CSF sont impliquées [4]. Le G-CSF stimule la production, l'activation, la maturation et le chimiotactisme des neutrophiles $[4,5]$. Il a été démontré que peu de temps avant 
l'apparition des symptômes, le taux sérique de G-CSF augmente de façon importante et diminue lentement durant la phase de guérison [6]. L'augmentation du taux de G-CSF donne probablement lieu à une augmentation du taux de neutrophiles périphériques et à l'infiltration neutrophilique au niveau du derme. Le G-CSF peut donc causer un SS chez des patients neutropéniques par induction de la prolifération des cellules souches, la différenciation des neutrophiles et la prolongation de la survie des neutrophiles [4]. L'intervalle entre l'administration de G-CSF et l'apparition des lésions cutanées varie de 3 jours à 1 semaine dans les cas décrits [4-6]. La fièvre et les lésions cutanées disparaissent, généralement sans séquelles, après l'arrêt de l'administration de G-CSF avec ou sans administration de corticostéroïdes [5].

\section{Conclusion}

Un diagnostic de syndrome de Sweet doit être considéré devant un tableau associant une éruption cutanée avec une infiltration neutrophilique, une fièvre, un échec de l'antibiothérapie et une réponse aux corticostéroïdes chez un patient ayant reçu du G-CSF.

\section{Déclaration de liens d'intérêts}

Les auteurs déclarent ne pas avoir de liens d'intérêts.

\section{Références}

[1] Paydas S. Sweet's syndrome: a revisit for hematologists and oncologists. Crit Rev Oncol Hematol 2013;86:85-95.

[2] Su WP, Liu HN. Diagnostic criteria for Sweet's syndrome. Cutis 1986;37:167-74.

[3] Walker DC, Cohen PR. Trimethoprim-sulfamethoxazole-associated acute febrile neutrophilic dermatosis: case report and review of drug-induced Sweet's syndrome. J Am Acad Dermatol 1996;34:918-23.

[4] Draper BK, Robbins JB, Stricklin GP. Bullous Sweet's syndrome in congenital neutropenia: association with pegfilgrastim. J Am Acad Dermatol 2005;52:901-5.

[5] Prevost-Blank PL, Shwayder TA. Sweet's syndrome secondary to granulocyte colony-stimulating factor. J Am Acad Dermatol 1996;35:995-7.

[6] Arbetter KR, Hubbard KW, Markovic SN, Gibson LE, Phyliky RL. Case of granulocyte colony-stimulating factor-induced Sweet's syndrome. Am J Hematol 1999;61:126-9.

[7] Machan M, Matthys B, Fraga GR. Pegfilgrastim-induced Sweet's syndrome: a case report. Int J Dermatol 2014;53:1275-7.

[8] Llamas-Velasco M, Garcia-Martin P, Sanchez-Pérez J, Fraga J, Garcia-Diez A. Sweet's syndrome with subcutaneous involvement associated with pegfilgrastim treatment: first reported case. J Cutan Pathol 2013;40:46-9.

[9] Nelson CA, Noe MH, McMahon CM, Gowda A, Wu B, Ashchyan HJ, et al. Sweet syndrome in patients with and without malignancy: a retrospective analysis of 83 patients from a tertiary academic referral center. J Am Acad Dermatol 2018;78:303-9. 